\title{
Fish Flavor
}

National Cancer Institute

\section{Source}

National Cancer Institute. Fish Flavor. NCI Thesaurus. Code C79134.

Similar to the terms LIVER and MEAT flavor, this is a broad category term that encompass the various types of FISH (e.g., salmon and tuna). 